\title{
Protein arginine methyltransferase 6-dependent gene expression and splicing: association with breast cancer outcomes
}

\author{
Dennis H Dowhan ${ }^{1 *}$, Matthew J Harrison ${ }^{1 *}+$, Natalie A Eriksson ${ }^{1 *}$, \\ Peter Bailey ${ }^{2 *}$, Michael A Pearen ${ }^{1}$, Peter J Fuller ${ }^{3}$, John W Funder ${ }^{3}$, \\ Evan $R$ Simpson ${ }^{3}$, Peter J Leedman ${ }^{4}$, Wayne D Tilley ${ }^{5}$, Melissa A Brown', \\ Christine L Clarke ${ }^{6}$ and George E O Muscat ${ }^{1}$
}

\footnotetext{
${ }^{1}$ Institute for Molecular Bioscience and ${ }^{2}$ School of Chemistry and Molecular Biosciences, The University of Queensland, Brisbane, Queensland 4072, Australia

${ }^{3}$ Prince Henry's Institute for Medical Research, Clayton, Victoria 3168, Australia

${ }^{4}$ Laboratory for Cancer Medicine, Western Australian Institute for Medical Research and University of Western Australia Centre for Medical Research, Royal Perth Hospital, Perth, Western Australia 6000, Australia

${ }^{5}$ Dame Roma Mitchell Cancer Research Laboratories, School of Medicine, Hanson Institute, The University of Adelaide, Adelaide, South Australia 5000, Australia

${ }^{6}$ University of Sydney Western Clinical School, Westmead Institute for Cancer Research, Westmead Millennium Institute,

Westmead, New South Wales 2145, Australia

(Correspondence should be addressed to D H Dowhan; Email: d.dowhan@uq.edu.au)

*(D H Dowhan, M J Harrison, N A Eriksson and P Bailey are co-first authors)

${ }^{\dagger}$ (M J Harrison is now at School of Biomedical Sciences, The University of Queensland, Brisbane, Queensland 4072, Australia)
}

\begin{abstract}
Protein arginine methyltransferase-6 (PRMT6) regulates steroid-dependent transcription and alternative splicing and is implicated in endocrine system development and function, cell death, cell cycle, gene expression and cancer. Despite its role in these processes, little is known about its function and cellular targets in breast cancer. To identify novel gene targets regulated by PRMT6 in breast cancer cells, we used a combination of small interfering RNA and exon-specific microarray profiling in vitro coupled to in vivo validation in normal breast and primary human breast tumours. This approach, which allows the examination of genome-wide changes in individual exon usage and total transcript levels, demonstrated that PRMT6 knockdown significantly affected i) the transcription of 159 genes and ii) alternate splicing of 449 genes. The PRMT6-dependent transcriptional and alternative splicing targets identified in vitro were validated in human breast tumours. Using the list of genes differentially expressed between normal and PRMT6 knockdown cells, we generated a PRMT6-dependent gene expression signature that provides an indication of PRMT6 dysfunction in breast cancer cells. Interrogation of several well-studied breast cancer microarray expression datasets with the PRMT6 gene expression signature demonstrated that PRMT6 dysfunction is associated with better overall relapse-free and distant metastasis-free survival in the oestrogen receptor (ER (ESR1)) breast cancer subgroup. These results suggest that dysregulation of PRMT6-dependent transcription and alternative splicing may be involved in breast cancer pathophysiology and the molecular consequences identifying a unique and informative biomarker profile.
\end{abstract}

Endocrine-Related Cancer (2012) 19 509-526

\section{Introduction}

Protein arginine methylation is a common posttranslational modification that was first described over 40 years ago (Paik \& Kim 1967). It is catalysed by a family of 11 human protein arginine methyltransferases (PRMTs) and results in the transfer of either one or two methyl groups from the methyl donor $S$-adenosyl-L-methionine to guanidino nitrogen atoms in arginine residues of the target protein. This alters the function and activity of the protein and 
impacts on a diverse range of cellular processes including differentiation, DNA repair, transcription, RNA processing, signal transduction, cell localisation and apoptosis (Bedford \& Richard 2005). As would be expected of proteins that regulate such diverse processes, their aberrant expression and activity have been linked to a number of human diseases, including cancer (Aletta \& Hu 2008).

PRMTs regulate transcription by functioning as co-activators for a range of transcription factors, including steroid/nuclear receptors, p53, YY1 and NF- $\kappa$ B; E2F1; MEF2C and $\beta$-catenin (Bedford \& Clarke 2009, Lee \& Stallcup 2009). We have recently shown that PRMT6 can also co-activate steroid hormone receptors (SHRs), including the ER $\alpha$, progesterone receptor and glucocorticoid receptor (GR (NR3C1)), in a methylation-dependent manner (Harrison et al. 2010). This appears to contrast with the studies demonstrating that PRMT6 can repress transcription by methylating histone 3 at arginine 2 (H3R2), which prevents the mixed lineage leukaemia methyltransferase complex binding to and methylating histone 3 at lysine 4 (H3K4; Guccione et al. 2007, Hyllus et al. 2007, Iberg et al. 2008). Therefore, whether PRMT6 functions as a transcriptional enhancer or repressor in relation to gene expression remains controversial.

PRMTs also regulate alternate splicing in a methylation-dependent manner. Co-activatorassociated arginine methyltransferase 1 (CARM1) can methylate a number of proteins involved in premRNA processing and promotes skipping of both a splicing reporter and the endogenous CD44 gene (Cheng et al. 2007). PRMT6, which can methylate the splicing factors RDA288 and heterogeneous nuclear ribonucleoprotein D-like (Cheng et al. 2007), promotes exon skipping of vascular endothelial growth factor (VEGF (VEGFA)) and spleen tyrosine kinase $(S Y K)$ genes in MCF-7 and T-47D breast cancer cells (Harrison et al. 2010).

Initial studies have identified several PRMT proteins as having a key role in SHR-mediated prostate cancer, breast cancer, gastric cancer, lymphoma and leukaemia (Teyssier et al. 2010). Many proteins involved in RNA splicing/processing are methylated and/or associated in complexes with PRMTs. The distinct possibility arises that PRMTs are able to directly methylate and regulate proteins involved in RNA processing events and alternative splicing. Exon profiling in the framework of transcriptomic analysis is an emerging area in disease diagnosis and prognosis (Dutertre et al. 2010). The fact that certain PRMTs are associated with RNA processing and gene expression makes them a clear choice to examine their ability to regulate alternative splicing and transcription in breast cancer.

\section{Materials and methods}

\section{Cell culture and transfection}

MCF-7 cells were maintained in DMEM nutrient mixture F-12 plus 10\% FBS. T-47D cells were maintained in RPMI-1640 medium plus 10\% FBS, $5 \mu \mathrm{g} / \mathrm{ml}$ insulin, $10 \mathrm{mM}$ HEPES and $1 \mathrm{mM}$ sodium pyruvate. Both breast cancer cells lines were maintained at $37^{\circ} \mathrm{C}$ and $5 \% \mathrm{CO}_{2}$. For small interfering RNA (siRNA) experiments in MCF-7 and/or T-47D cell lines, cells were transfected for $48 \mathrm{~h}$ with PRMT6specific siRNA or control siRNA duplexes at a final concentration of $10 \mathrm{nM}$ using RNAiMAX (Invitrogen, Victoria, Australia), with six biological replicates for each treatment regime, as described previously (Harrison et al. 2010). siRNA sequences were PRMT6-siRNA-1, sense 5'-CGGGACCAGCUGUACUACGTT-3', PRMT6-siRNA-1, antisense 5'-CGUAGUACAGCUGGUCCCGTT-3', PRMT6-siRNA-2, sense 5'-GCACUUGUAUUUCCGUAUATT-3', PRMT6-siRNA-2, antisense 5'-UAUACGGAAAUACAAGUGCTT- $3^{\prime}$. A siRNA control, Silencer Select negative control \#1 (Ambion, Texas, USA), was used as a negative control (control siRNA), which has a proprietary unreleased sequence.

\section{Exon microarray data analysis}

Labelling and hybridisation of the Affymetrix Exon 1.0 ST Arrays were performed by the Ramaciotti Centre for Gene Function Analysis (UNSW, Sydney, Australia) following the recommended Affymetrix protocols. Exon array data were analysed using XRAY excel array expression analysis software (Biotique Systems, Inc., Nevada, USA). Normalized data and raw gene expression files have been submitted to the NCBI Gene Expression Omnibus (GEO; Barrett et al. 2005), accession number: GSE36542.

Input files were normalised with full quantile normalisation to remove variation produced amongst samples during array labelling, hybridisation and scanning. Principal component analysis was performed using GeneSpring GX version 11 (Agilent, Victoria, Australia) on each sample to confirm similarity between samples with the same treatment. Principal component analysis scores were used to form a three-dimensional plot of each sample.

Probe sets then underwent filtering (to ensure that only high quality probe sets were included in the 
analysis), GC content-based probe screening, log transformation and background correction using GC-matched probes. Individual probe sets that are not significantly expressed above background can produce false positives for alternative splicing and so were excluded from tests of alternative exon usage. Probe sets are ranked as Core, Extended or Full depending on their reliability. Only Core probe sets, which are derived from high-quality genomic features like RefSDefault (www.ncbi.nlm.nih.gov) or Ensembl (www.ensembl.org), were used in the analysis. The expression score for each probe set was then derived via Tukey's median-polish and Mixed Model, and Nested ANOVA was then used to identify changes in gene expression or alternative splicing between groups. Alternative splicing results were corrected using the Benjamini and Hochberg False Discovery Rate to remove false positives. Genes were considered to be differentially expressed or alternatively spliced when $P<0.01$ (Supplementary Tables 2 and 3).

To determine splice events within significantly alternatively spliced genes, probe sets showing 'nonparallel' lines when average group expression is plotted over the probe sets were identified. Probe sets displaying 'non-parallel' expression were assigned to a particular exon using X:map (http://xmap.picr.man.ac. $\mathrm{uk} /$ ). Changes in exon-specific splicing were then compared to known alternative splicing events using X:map (built using the Ensembl database (www.ensembl.org)) and FAST DB (http://www.fastdb.com/fastdb2/frame.html), built by combining databases within www.ensembl.org, http://genome.ucsc. edu and www.ncbi.nlm.nih.gov.

\section{RNA isolation, reverse transcription and quantitative real-time PCR}

Total RNA was extracted from cells using Trizol Reagent (Invitrogen) and purified using RNeasy RNA columns, with on-column DNase treatment (Qiagen, Victoria, Australia). RNA was normalised using u.v. spectrophotometry and agarose gel electrophoresis. RNA integrity was determined using RNA 6000 Nano chips (Agilent) on a 2100 BioAnalyser (Agilent) according to the manufacturer's instructions. cDNA was synthesized from $600 \mathrm{ng}$ of total RNA using TaqMan reverse transcription reagents, according to the manufacturer's instructions (Applied Biosystems, Victoria, Australia).

Gene expression levels were determined by 384-well quantitative real-time PCR (Q-RT-PCR) on a 7900HT Fast RT-PCR System (Applied
Biosystems) as described previously (Harrison et al. 2010). RT-PCR was performed using Assay on Demand TaqMan primer/probes (Applied Biosystems) or Sybr green; PRMT6 (Hs00250803_s1) CARM1 (Hs00406354_m1), PRMT1 (Hs01587651_g1), NCOA4 (Hs00428331_g1), FKBP4 (Hs00427038_g1), HIPK3 (Hs00178628_m1), ARL6IP1 (Hs00760013_s1), YWHAE (Hs00356749_g1), MINA (Hs00262155_m1), PTEN (Hs02621230_s1), IGFBP3 (Hs00181211_m1), THEM4 (Hs00940013_g1), BRCA2 and CDKN1A interacting protein $\alpha(\mathrm{BCCIP} \alpha$; Hs01567098_m1) and BCCIP $\beta$ (Hs01555220_m1). All expression levels were normalised to $18 S$ RNA or RLP0 expression levels using TaqMan primer/probe sets (Applied Biosystems). Target cDNA levels were analysed by Q-RT-PCR in $10 \mu \mathrm{l}$ reactions using TaqMan PCR master mix (Applied Biosystems), TaqMan probe/primer sets and cDNA (5\% of the starting $600 \mathrm{ng}$ of RNA). PCR was initiated at $95^{\circ} \mathrm{C}$ for $10 \mathrm{~min}$ to activate Amplitaq Gold DNA polymerase followed by 45 cycles of $95^{\circ} \mathrm{C}$ for $15 \mathrm{~s}$ and $60{ }^{\circ} \mathrm{C}$ for a $1 \mathrm{~min}$ two-step thermal cycling. Relative changes in gene expression were calculated using the $\Delta \Delta C_{\mathrm{t}}$ method. For each experiment, six replicate wells of cells were used for each experimental condition and all experiments were repeated twice.

Alternative splicing validation of ninein (GSK3B interacting protein) (NIN), catenin (cadherinassociated protein)-delta 1 (CTNND1) and thioesterase superfamily member 4 (THEM4) was performed by Q-RT-PCR on a 7900HT Fast RT-PCR System (Applied Biosystems) in a $10 \mu \mathrm{l}$ reaction using SYBR-green master mix (Applied Biosystems) using manufacturer's instructions. Primers, which were designed to specifically amplify the alternatively spliced isoforms, were NIN inclusion forward 5'-GAGTCACTATTTTAGTTAAGC- ${ }^{\prime}$, NIN inclusion - reverse $5^{\prime}$-AGATTCATATCTCAGAAGTTC-3', NIN skipping - forward 5'-GAACTAGAGCAGTTTCACCAG- $3^{\prime}$, NIN skipping - reverse 5'-CAGATTCATATCTTCCCTCCT-3', CTNND1 inclusion - forward 5'-ACACCCTTGATGCAGGACGAG-3', CTNND1 inclusion - reverse 5'-AATCTTCTGCATGGAGGGGTA-3', CTNND1 skipping - forward 5'-ACAACTATTCCACACCAAATG-3', CTNND1 skipping - reverse 5'-AATCTTCTGCATCAAGGGTGT-3 ${ }^{\prime}$, THEM4 inclusion - forward 5'-CATTTTCTTGCCTGGTTAATG-3', THEM4 inclusion - reverse $5^{\prime}$-GTTGGTCAGGCTGGTCTTGAA-3' and THEM4 skipping - forward 5'-ACAAGGACCTAAGACTGCTCT-3', THEM4 skipping - reverse $5^{\prime}$-AAGCTTTGGGTCAAGAAAATG-3 ${ }^{\prime}$. RT-PCR was initiated at $95^{\circ} \mathrm{C}$ for $10 \mathrm{~min}$ to activate Amplitaq Gold DNA polymerase followed 
by 45 cycles of $95{ }^{\circ} \mathrm{C}$ for $15 \mathrm{~s}$ and $56^{\circ} \mathrm{C}$ for a $1 \mathrm{~min}$ two-step thermal cycling. Relative changes in gene expression were calculated using the $\Delta \Delta C_{\mathrm{t}}$ method. For each experiment, six replicate wells of cells were used for each experimental condition and all experiments were repeated twice.

Melt curve analysis was performed to ensure that only one product was amplified in the Q-RT-PCRs. To determine the specificity of the amplified product, PCR fragments amplified from MCF-7 cell cDNA using the above primer sets were sub-cloned into pCR2.1 using the TOPO-TA cloning kit (Invitrogen) and sequenced. We also verified that the Q-RT-PCR assay produces a linear standard curve by plotting the log of the starting quantity of the template against the $C_{\mathrm{t}}$ value after serially diluting the pCR2.1-cloned PCR products and analysing them by Q-RT-PCR using the appropriate primers. We also verified that primer sets designed to detect inclusion do not detect the pCR2.1-cloned skipping spliced product, and primer sets designed to detect skipping do not detect the pCR2.1-cloned PCR product including the variable exon in Q-RT-PCR assays. Statistical analysis of RT-PCR data was performed using either one- or two-tailed Student's $t$-tests as appropriate.

\section{Human breast tissue samples}

Data and de-identified breast tissue samples (either fresh frozen tissue or purified total RNA) were obtained from the tissue banks listed in the Acknowledgements section. The breast tissue samples used were from individual cases of primary invasive ductal carcinoma (IDC) or normal breast samples, with no known history of breast disease, were collected following breast reduction mammoplasty or from women who had volunteered normal breast biopsy tissue. This study was approved by the human research ethics committees of all participating institutions.

\section{Q-RT-PCR of human breast tissue samples}

Commercial micro-fluidic cards, the TaqMan LowDensity Arrays (TLDA, Applied Biosystems) and Q-RT-PCR using 384-well 7900HT Fast RT-PCR System were used to profile gene expression for breast tissue samples. The geNorm software imbedded within the ABI/intergromics StatMiner V4.1 software package was used to compute least expression variation and select the most appropriate, stable and robust combination of internal control genes with which to normalise the expression data (against the mean of the most stable controls). TLDAs were used according to the manufacturer's instructions and analysed as described previously
(Myers et al. 2009, Raichur et al. 2010). The TaqMan Gene Expression Assays used as controls were MRPL19 (Hs00608519_m1), PGK1 (Hs99999906_m1), PPIA (Hs99999904_m1), TFRC (Hs99999911_m1) and UBC (Hs00824723_m1) median. The TaqMan Gene Expression Assays used were AKR1C2;AKR1C1 (Hs00413886_m1), SLIRP (Hs00364015_m1), EDF1 (Hs00610152_m1), FKBP4 (Hs00427038_g1), HIPK3 (Hs00178628_m1), NCOA4 (Hs00428328_m1), NQO1 (Hs00168547_m1), PA2G4 (Hs00854538_g1), PRKCD (Hs00178914_m1), PTEN (Hs00829813_s1) and TRIP13 (Hs00188500_m1). For each sample, $1.5 \mu \mathrm{g}$ of total RNA was reverse transcribed using random hexamers with SuperScript III reverse transcriptase (Invitrogen) in a total volume of $45 \mu \mathrm{l}$. A volume of DNase/RNase-free water and an equal volume of TaqMan universal master mix was added to each TLDA fill reservoir. Each GCT sample was run once whilst the cell lines were run three times. Four reservoirs per sample were filled. The TLDA includes all gene assays and endogenous controls in triplicate. After sealing the plate, it was run on an ABI 7900HT Real-Time instrument.

Examination of alternative splicing in breast tissue samples was done by 384-well Q-RT-PCR as described earlier, using IPO8 (Hs00183533_m1) and TBP (Hs00427620_m1) median as the controls for that specific cohort of samples. TaqMan primer/ probes (Applied Biosystems) used were BCCIP $\alpha$ (Hs01567098_m1), ВCCIP $\beta$ (Hs01555220_m1), NINEIN-inclusion - forward: 5'-CACGTGCAGTGAGATGCAGC-3', NINEIN-inclusion - reverse: $5^{\prime}$ CTTCCTGAGATCCATTTAATGTCC-3', NINEINinclusion-Probe: 5'-6-FAM-TGAACTTCTGAGATATGAAT-MGB-3', NINEIN-skipping - forward: 5'-GAGCTCTTGGAAAAGCACCAA-3', NINEINskipping - reverse: 5'-CTTCCTGAGATCCATTTAATGTCC-3', NINEIN-skipping-Probe: 5'-6-FAMCAGGAGGGAAGATATGAATC-MGB-3', THEM4inclusion - forward: $5^{\prime}$-ACACCTACTGAATGGATTCAAGACT-3', THEM4-inclusion - reverse: $5^{\prime}$-AGGGATCCGCCTGCCTTG-3', THEM4-inclusion-Probe: 5'-6-FAM-CATTTTCTTGCCTGGTTA-MGB-3', THEM4-skipping - forward: 5'-ACGTACACCTACTGAATGGATTC-3', THEM4-skipping - reverse: 5'-CTGGGCCTGTGACATTTGTTC-3', THEM4skipping-Probe: 5'-6-FAM-CATTTTCTTGACCCAAAGC-MGB-3'. TaqMan primer/probes (Applied Biosystems) for $S Y K$ have been described previously (Harrison et al. 2010).

The TLDAs and Q-RT-PCR of breast tissue samples were analysed using the relative quantification method of $C_{\mathrm{t}}$ and the normalised $C_{\mathrm{t}}$ values $\left(\Delta C_{\mathrm{t}}\right)$ of the 
replicates between the control/calibrator and the target sample (normal breast) were compared as described previously (Myers et al. 2009, Raichur et al. 2010).

\section{Analysis of breast cancer microarray datasets}

Statistical analysis was performed in R (http://www. r-project.org/). Gene expression datasets were retrieved from Bioconductor (http://bioconductor. org). The gene signature used comprised the 159 genes that were differentially expressed (i.e. up- and down-regulated) in a significant manner relative to controls $(\geq 1.3$-fold, $P<0.01)$ as identified in exon array analysis (Supplementary Table 2, see section on supplementary data given at the end of this article). Genes listed in Supplementary Table 2 that did not have a fold change $\geq 1.3$ but did have a probability of differential expression $P<0.01$ (highlighted in grey) were not included in the signature. Experimental data packages examined include BreastCancerUPP (Miller et al. 2011), BreastCancerNKI (van de Vijver et al. 2002) and BreastCancerTRANSBIG (Desmedt et al. 2008). PRMT6 signature scores were computed using the sig.score function from the Genefu package (Haibe-Kains et al. 2010a). This function computes a signature score from a gene list (aka gene signature), i.e. a signed average as previously published (Sotiriou et al. 2006, Haibe-Kains 2009). Briefly, positive or high signature scores are indicative of PRMT6 dysfunction (and by inference low PRMT6 expression levels), whereas negative or low signature scores are indicative of normal PRMT6 function (and by inference high PRMT6 levels). Survival analysis was performed using the SurvComp package (Haibe-Kains et al. 2008, 2010b). PRMT6 signature scores were split according to their $33 \%$ and $66 \%$ quantiles. Upper and lower quantiles were used for survival analysis. We considered relapse-free survival (RFS) as the survival endpoint. When RFS was not available, we used distant metastasis-free survival (DMFS) data. All survival data were censored at 10 years. Survival curves were based on Kaplan-Meier estimates.

\section{Results}

\section{siRNA depletion of PRMT6 and detection of genome-wide exon expression}

MCF-7 breast cancer cells were transfected with a validated siRNA targeting PRMT6 (PRMT6-siRNA-1) or a control siRNA, as previously reported (Harrison et al. 2010). PRMT6 siRNA-1 specifically reduced mRNA levels of PRMT6 by $\sim 80 \%$ but did not affect the levels of PRMT1 or CARM1 (Fig. 1A). Alterations in gene transcription and alternative splicing were determined by hybridisation of labelled cDNA onto GeneChip Human Exon 1.0 ST Arrays (Affymetrix). Following full quantile normalisation, principal component analysis was performed, with exon arrays clustering into groups depending on the treatment regime (Fig. 1B). This result demonstrates that replicates of the same siRNA treatment produced a similar exon expression profile but a distinctly different profile in relation to the siRNA treatment based on clustering distance. The data were filtered by removing less reliable probes, probes with a low or high GC content, and probes that were not significantly expressed above background. The effects of the filtering are summarised in Supplementary Table 1, see section on supplementary data given at the end of this article. Of the different alternatively spliced products (referred to as transcript clusters) available for analysis following background correction, 3316 were expressed in both treatment groups.

A

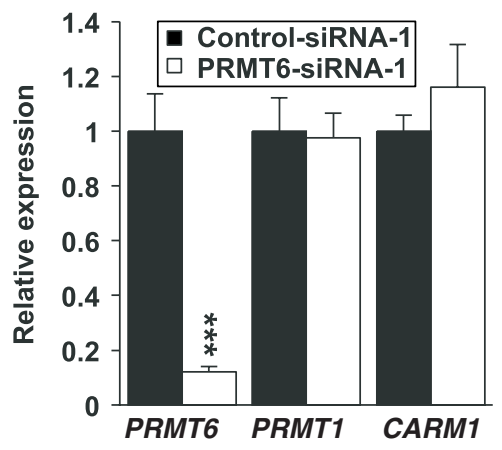

B

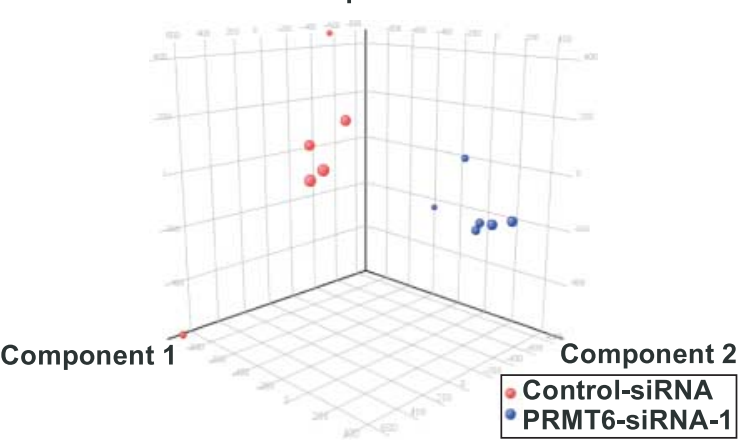

Figure 1 PRMT6 knockdown and exon array analysis validation. (A) MCF-7 cells were transfected with control siRNA or PRMT6 siRNA-1 for $48 \mathrm{~h}$. RNA was harvested and analysed for expression of PRMT6, PRMT1 and CARM1 by TaqMan Q-RT-PCR. Results were normalised to expression levels following transfection with control siRNA. Each data point represents the mean and S.D. of six transfected cultures. ${ }^{\star * \star} P<0.001$. (B) Graph of three-dimensional principal components analysis scores, with analysis performed on each sample. 
A
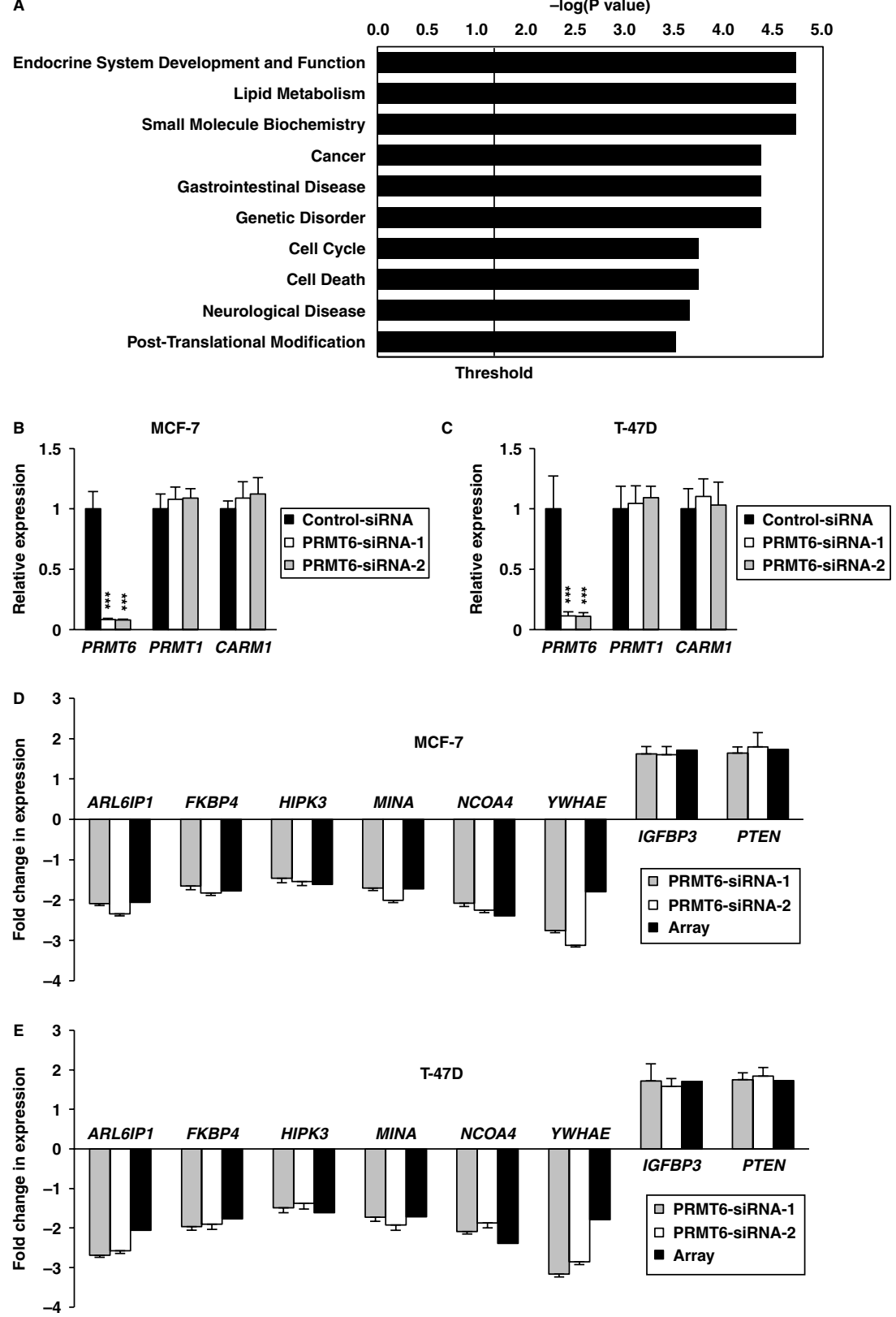

Figure 2 PRMT6 promotes and represses transcription. (A) Ingenuity analysis of exon array data following PRMT6 knockdown, showing the top ten pathways regulated by PRMT6 at the transcriptional level. (B) MCF-7 cells were transfected with control siRNA, PRMT6 siRNA-1 or PRMT6 siRNA-2 for $48 \mathrm{~h}$. RNA was harvested and analysed expression of PRMT6, PRMT1 and CARM1 by TaqMan Q-RT-PCR. Results were normalised to expression levels following transfection with control siRNA. (C) T-47D cells were transfected with control siRNA or PRMT6 siRNA-1 for $48 \mathrm{~h}$. RNA was harvested and analysed to examine the expression of PRMT6, PRMT1 and CARM1 by TaqMan Q-RT-PCR. Results were normalised to expression levels following transfection with control siRNA. (D) MCF-7 cells were transfected with control siRNA, PRMT6 siRNA-1 or PRMT6 siRNA-2 for $48 \mathrm{~h}$. RNA was harvested and analysed by TaqMan Q-RT-PCR for expression of ARL6IP1, FKBP4, HIPK3, MINA, NCOA4, YWHAE, IGFBP3 or PTEN. Included are gene expression changes observed in the exon arrays (Array). Results are shown as fold change in expression compared with control siRNA values. (E) T-47D cells were transfected with control siRNA-1, PRMT6 siRNA-1 or PRMT6 siRNA-2 for $48 \mathrm{~h}$. RNA was harvested and analysed by TaqMan Q-RT-PCR for expression of ARL6IP1, FKBP4, HIPK3, MINA, NCOA4, YWHAE, IGFBP3 or $P T E N$. Included are gene expression changes observed in the exon arrays (Array). Results are shown as fold change in expression compared with control siRNA values. In graphs B, C, D and E, each data point represents the mean and s.D. of six transfected cultures. ${ }^{* \star} P<0.001$ compared to respective control. Results shown are from a single experiment, which is representative of two independent experiments. 


\section{PRMT6 influences both gene activation and repression}

Analysis from genome-wide exon expression profiling of whole transcripts identified 159 genes that had a fold change $\geq 1.3$ and were significantly differentially expressed compared to controls $(P<0.01$; Supplementary Table 2). Of these 159 genes, 121 were downregulated and 38 were up-regulated in response to PRMT6 knockdown. This demonstrated that attenuation of PRMT6 expression resulted in the activation and repression of downstream gene expression. The differentially expressed genes were analysed by Ingenuity Pathway Analysis in order to identify functional groups regulated by PRMT6. Several functional groups were found to be over-represented, including endocrine system development and function, small molecule biochemistry, cell death and cancer, indicating that PRMT6 may regulate these processes (Fig. 2A).

In order to validate the gene expression results obtained from the exon array, eight genes were chosen for re-examination by Q-RT-PCR in the $\mathrm{ER} \alpha+\mathrm{MCF}-7$ and T-47D cell lines. These genes, taken from the list of 159 differentially expressed genes, comprised six genes with decreased expression and two with increased expression following PRMT6 knockdown. The genes were chosen for their biological significance and had relevance to either steroid hormone-dependent gene expression (FK506 binding protein 4, $59 \mathrm{kDa}$ : FKBP4; homeodomain interacting protein kinase 3: HIPK3 and nuclear receptor co-activator 4: NCOA4) (Yeh \& Chang 1996, Moilanen et al. 1998, Riggs et al. 2003) and/or cancer (NCOA4, ADP-ribosylation factor-like 6 interacting protein 1: ARL6IP1; MYC-induced nuclear antigen: MINA; tyrosine 3-monooxygenase/tryptophan 5-monooxygenase activation protein, epsilon polypeptide: YWHAE; phosphatase and tensin homolog: PTEN and insulin-like growth factor binding protein 3: IGFBP3) (Eng 2003, Hu et al. 2004, Teye et al. 2004, Cimino et al. 2008, Peng et al. 2008, Guo et al. 2010, Ingermann et al. 2010). PRMT6 levels were decreased in MCF-7 or T-47D breast cancer cells by siRNA for $48 \mathrm{~h}$. We conducted further validation, utilising two (different) non-overlapping siRNAs targeting PRMT6 (individually) to decrease the likelihood of observing non-specific off-target effects. These two siRNAs have been previously validated and do not affect PRMT1 or CARM1 RNA levels (Fig. 2B and C; Harrison et al. 2010). Q-RT-PCR following knockdown of PRMT6 with both siRNAs gave similar results to the exon array, with the expression of all eight genes approximating the fold changes observed in the exon array in both MCF-7 and T-47D cells (Fig. 2D and E).

\section{PRMT6-dependent alternative splicing}

Analysis of genome-wide exon expression profiling of individual exons identified 449 genes as being alternatively spliced compared with controls after multiple testing correction with the Benjamini and Hochberg False Discovery Rate $(P<0.01$; Supplementary Table 3). Analysis identified that 91 of these genes were also significantly differentially expressed, demonstrating that PRMT6 can regulate both transcription and pre-mRNA processing of the same gene (Fig. 3A). The 449 differentially alternatively spliced genes identified in the array were analysed by Ingenuity Pathway Analysis to determine cellular pathways that may be regulated by PRMT6-dependent alternative splicing. Several pathways were over-represented, including cell death, cell cycle and cancer (Fig. 3B).

In order to validate the alternative splicing array data, four genes that indicated differential exon
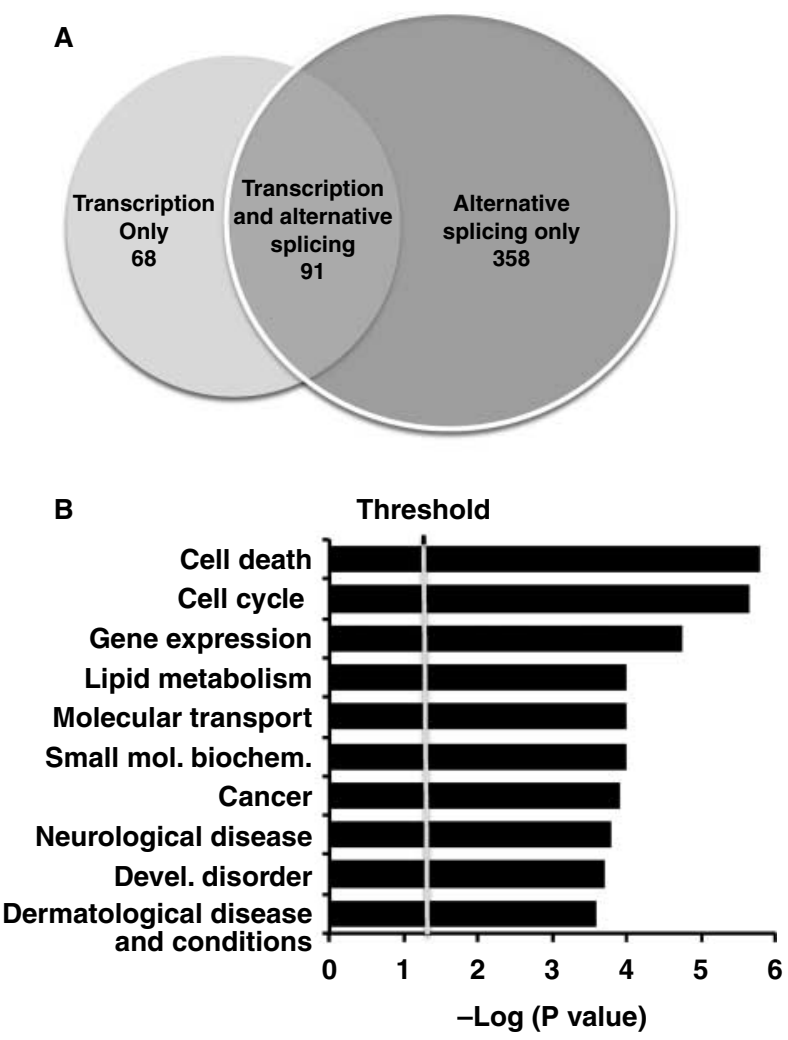

Figure 3 PRMT6 regulates alternative splicing. (A) Diagrammatic representation of the number of genes identified in the exon array that are regulated by PRMT6 at the levels of transcription, alternative splicing or both. (B) Ingenuity analysis of exon array data following PRMT6 knockdown, showing the top ten pathways regulated by PRMT6 at the level of alternative splicing. 
alternative splicing events were chosen from the list of 449 alternatively spliced genes by their biological relevance to cancer, namely NIN, CTNND1, THEM4 and BCCIP. NIN plays a role in the cell cycle and is involved in preventing microtubule aster-organisation defects during microtubule nucleation (Abal et al. 2002, Dammermann \& Merdes 2002, Stillwell et al. 2004). CTNND1 is thought to have a metastatic promoting role in breast cancer (van Roy \& McCrea 2005), THEM4 is involved in apoptosis (Parcellier et al. 2009) and BCCIP is involved with inhibiting breast cancer cell growth (Meng et al. 2004). The details of the genes chosen for validation are shown in Table 1. PRMT6 knockdown coupled with exon-specific Q-RT-PCR was used to examine the alternative splicing of these genes. Results are described in the sections below.

\section{PRMT6-dependent regulation of NIN exon skipping}

Comparison of probe sets within the NIN gene indicates that NIN may be alternatively spliced following PRMT6 knockdown. Seven probe sets (probe sets 24-30) displayed higher than expected expression following PRMT6 knockdown, indicative of potential differential alternative splicing (Fig. 4A). The NIN gene is composed of 34 exons, and all 7 'non-linear' probe sets map to exon 19, indicating that knockdown of PRMT6 leads to preferential inclusion of exon 19. Two transcripts within the Ensembl database display skipping of NIN exon 19 (ENST00000389868 and ENST00000382043), and this underscores alternative splicing of exon 19 and is an identified biologically relevant RNA processing event.

Table 1 Genes analysed by Q-RT-PCR to validate differential alternative splicing observed in exon array. The $P$ value (corrected) is the probability of differential alternative splicing (corrected by the Benjamini and Hochberg False Discovery Rate), and rank relates to the position of the gene amongst all genes in the array when ranked for $P$ value (corrected)

\begin{tabular}{|c|c|c|c|}
\hline $\begin{array}{l}\text { Gene } \\
\text { symbol }\end{array}$ & Gene name & $\begin{array}{l}\boldsymbol{P} \text { value } \\
\text { (corrected) }\end{array}$ & Rank \\
\hline NIN & $\begin{array}{l}\text { NINEIN (GSK3B } \\
\text { interacting protein) }\end{array}$ & $2.1 \times 10^{-34}$ & 2 \\
\hline CTNND1 & $\begin{array}{l}\text { Catenin (cadherin- } \\
\text { associated protein) delta } 1\end{array}$ & $4.03 \times 10^{-14}$ & 10 \\
\hline BCCIP & $\begin{array}{l}\text { BRCA2 and CDK1NA } \\
\text { interacting protein }\end{array}$ & $3.18 \times 10^{-8}$ & 52 \\
\hline THEM4 & $\begin{array}{l}\text { Thioesterase superfamily } \\
\text { member } 4\end{array}$ & $1.09 \times 10^{-7}$ & 60 \\
\hline
\end{tabular}

In order to validate the regulation of NIN alternative splicing by PRMT6, we designed primers to detect inclusion and skipping of NIN exon 19 for use in Q-RT-PCR (Fig. 4B). Following reduction of PRMT6 levels by PRMT6-siRNA-1 or PRMT6-siRNA-2 in MCF-7 and T-47D breast cancer cells, we saw $\sim 3$ - to 3.3-fold increase in inclusion and a $\sim 2$.3-fold decrease in skipping of NIN exon 19, leading to an increase in the inclusion: skipping ratio of approximately sevenfold (Fig. 4C, D, E and F). This is in agreement with the increase in inclusion of NIN exon 19 observed in the exon array following PRMT6 knockdown and confirms that a cellular function of PRMT6 is to promote NIN exon 19 skipping.

\section{PRMT6 regulates a variety of alternative splicing events}

Examination of median probe set scores from the exon expression profiling indicates that PRMT6 regulates the inclusion of CTNND1 exon 20, alternative splicing of exon 3 and overall transcript levels of THEM4, as well as BCCIP isoform production by alternative terminal exon choice (Supplementary Figures 1A, 2A, $\mathrm{B}$ and $3 \mathrm{~A}$, see section on supplementary data given at the end of this article). The alternative splicing of specific exons within the CTNND1, THEM4 and $B C C I P$ genes identified from the exon array is supported by alternative transcripts present in the Ensembl databases. This indicates that these alternative splicing events are common and previously identified biologically relevant RNA processing events.

We designed Q-RT-PCR assays to detect these various alternative splicing events for CTNND1, THEM4 and BCCIP in the MCF-7 and T-47D breast cancer cell lines (Supplementary Figures 1B, 2C and 3B). Knockdown of PRMT6 and Q-RT-PCR analysis confirmed a $\sim 2$.2-fold increase in skipping of CTNND1 exon 20, a >1.5-fold decrease in expression and preferential inclusion of THEM4 exon 3 an approximate twofold increase in the BCCIP $\beta$ and a concomitant twofold decrease in the levels of BCCIP $\alpha$ (Fig. 5A, B and C). These results show significant changes in the alternative splicing of specific exons within the CTNND1, THEM4 and BCCIP genes (Supplementary Figures 1C, D, E, 2D, $\mathrm{E}, \mathrm{F}$ and $3 \mathrm{C}, \mathrm{D}$ and $\mathrm{E})$. This indicates that one cellular function of PRMT6 is to influence a variety of selective alternative splicing events, which may have pathophysiological consequences in several cancer-related signalling pathways. 


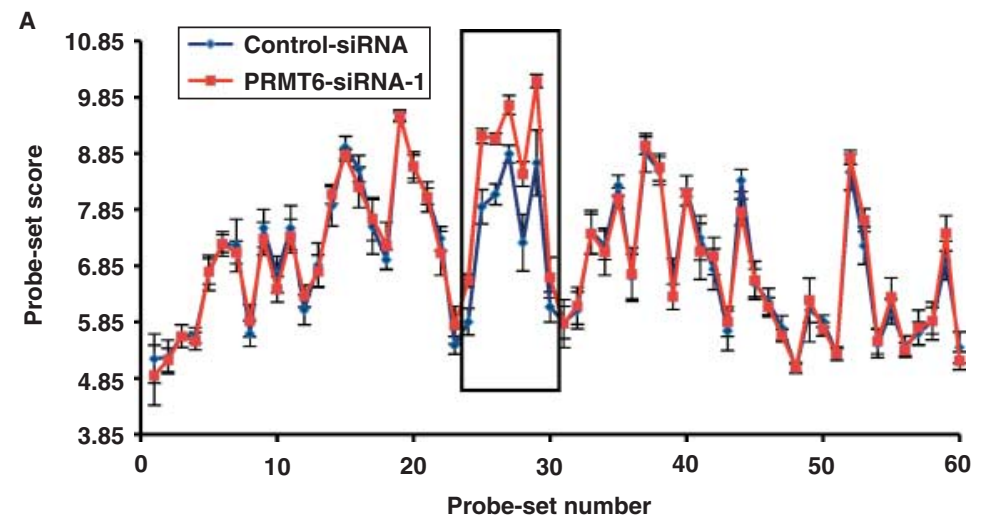

B

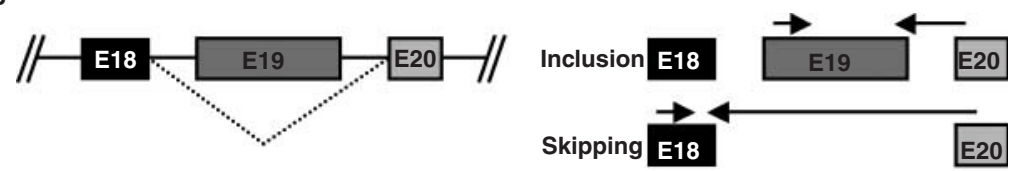

C

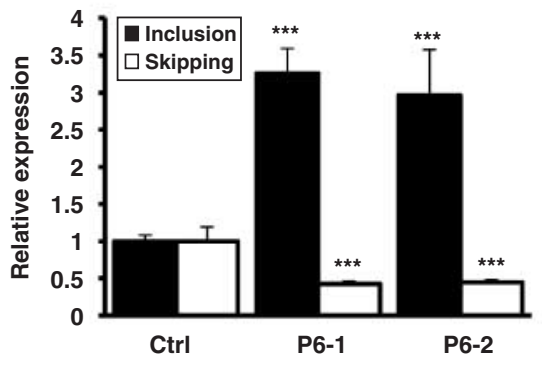

E

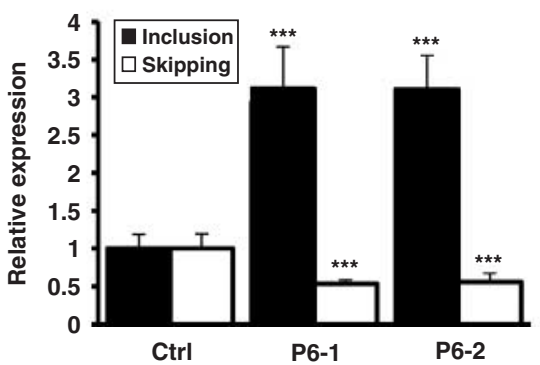

D

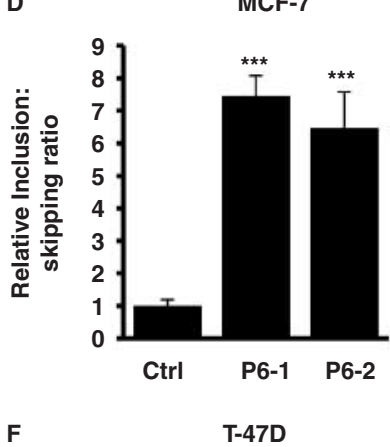

$\mathbf{F}$

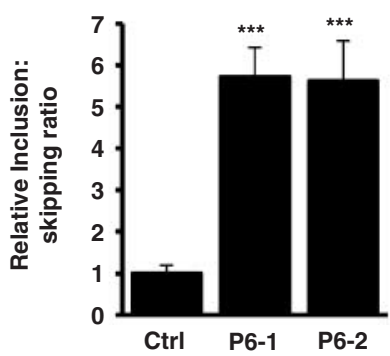

Figure 4 PRMT6 regulates alternative splicing of NIN exon 19. (A) MCF-7 cells were transfected with control siRNA or siRNA targeting PRMT6 for $48 \mathrm{~h}(n=6)$. RNA was harvested and hybridised to a Human $1.0 \mathrm{ST}$ exon array, and data were analysed using XRAY software. Knockdown of PRMT6 leads to 'non-linear' expression of probe sets 24-30 (highlighted by box). Data points show the mean and S.D. of probe set scores from each treatment group. (B) Q-RT-PCR method to detect exon 19 inclusion and skipping of the Ninein gene. To detect inclusion of exon 19, a forward primer within exon 19 and a reverse primer spanning the exon 19/20 boundary were used. To detect skipping, a forward primer within exon 18 and a reverse primer spanning the exon 18/20 boundary were used. (C) MCF-7 cells were transfected with control siRNA (Ctrl), PRMT6 siRNA-1 (P6-1) or PRMT6 siRNA-2 (P6-2) for 48 h. RNA was harvested and assayed for Ninein exon 19 inclusion and skipping by Q-RT-PCR. Inclusion and skipping expression levels were normalised to levels following treatment with control siRNA. (D) The relative inclusion:skipping ratio following PRMT6 knockdown in MCF-7 cells was calculated by dividing the relative inclusion expression by the relative skipping expression detected as in (C) and normalising to the control ratio. (E) T-47D cells were transfected with control siRNA (Ctrl), PRMT6 siRNA-1 (P6-1) or PRMT6 siRNA-2 (P6-2) for $48 \mathrm{~h}$ and analysed as in (C). (F) The relative inclusion:skipping ratio following PRMT6 knockdown in $\mathrm{T}-47 \mathrm{D}$ cells was calculated by dividing the relative inclusion expression by the relative skipping expression detected as in (E) and normalising to the control ratio. In graphs $C, D, E$ and F, each data point represents the mean and S.D. of six transfected cultures. ${ }^{* * *} P<0.001$ compared to respective control. Results shown are from a single experiment, which is representative of two independent experiments. 


\section{Differential expression of PRMT6 transcriptional and alternatively spliced genes in primary breast cancer tumours}

We assessed the expression of the mRNA encoding PRMT6 in a primary breast IDC cohort $(n=66, \mathrm{ER}+$ and $\mathrm{ER}-$ ) relative to a normal breast tissue cohort

A

CTNND1

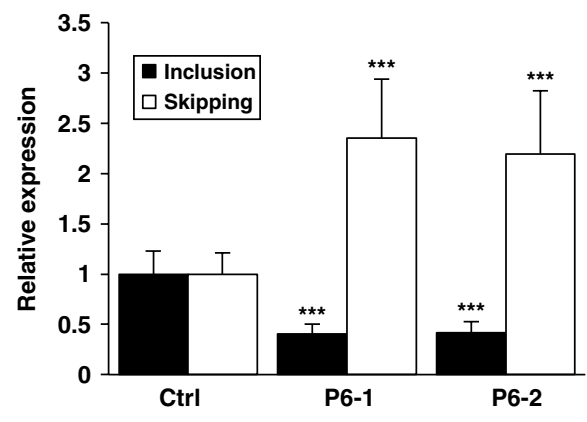

B

THEM4

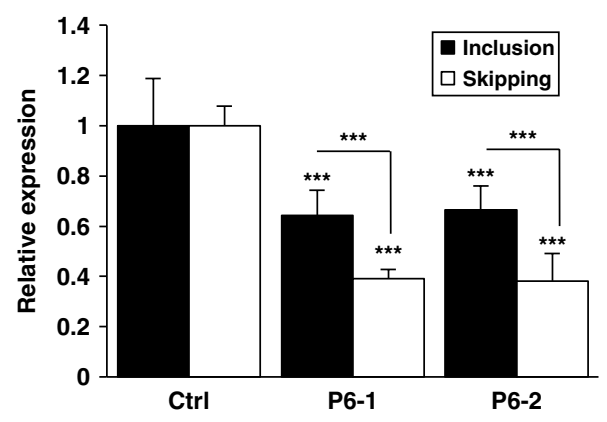

C

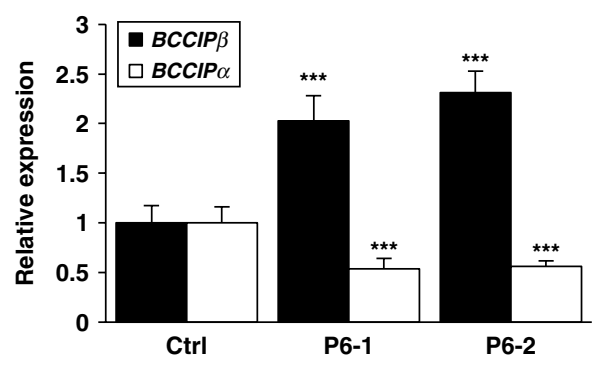

Figure 5 PRMT6 regulates multiple aspects of alternative splicing. MCF-7 cells were transfected with control siRNA (Ctrl), PRMT6 siRNA-1 (P6-1) or PRMT6 siRNA-2 (P6-2) for $48 \mathrm{~h}$ and RNA was harvested. (A) The corresponding cDNA was assayed for (A) CTNND1 exon 20 inclusion and skipping, (B) THEM4 exon 3 inclusion and skipping and (C) BCCIP $\alpha$ and $B C C I P \beta$ mRNA transcript levels by Q-RT-PCR, according to Supplementary Figures 1,2 and 3. Expression levels of specific mRNA transcripts to examine transcription, exon skipping, exon inclusion or specific mRNA isoform production were normalised to mRNA transcript levels following treatment with control siRNA. In graphs A, B and $C$, each data point represents the mean and S.D. of six transfected cultures. ${ }^{* \star} P<0.001$ compared to respective control. Results shown are from a single experiment, which is representative of two independent experiments.
A

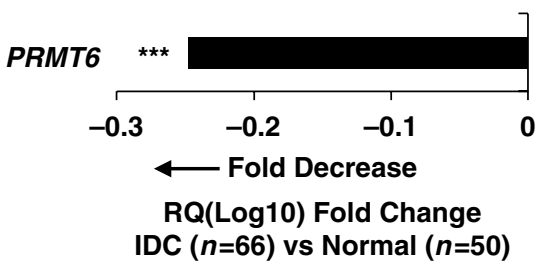

B

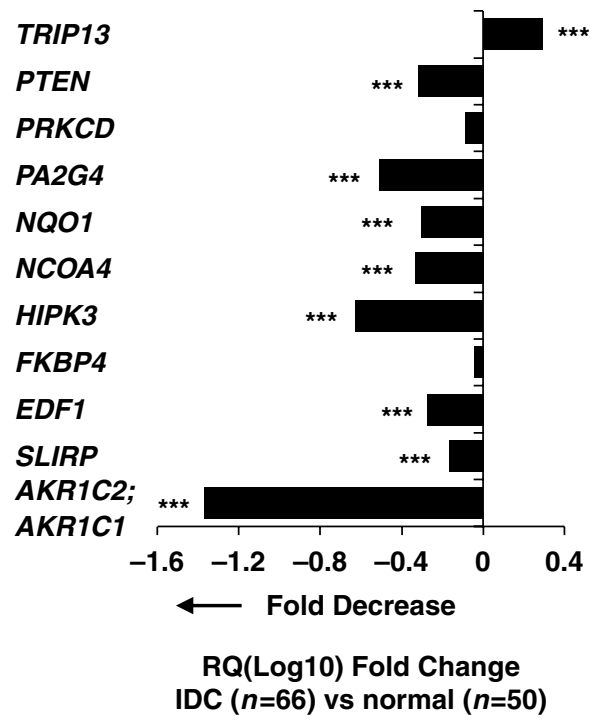

Figure 6 Expression of PRMT6 and associated transcriptional targets in breast cancer. (A) The expression of PRMT6 and

(B) PRMT6 transcriptionally regulated genes in invasive ductile carcinoma (IDC) human breast tissue samples $(n=66)$ relative to normal human breast tissue $(n=50)$. Data are presented as relative quantification (RQ) (log10) of genes from IDC human breast tissue samples relative to normal human breast tissue. Statistical analysis was conducted with Integromics Statminer software package, using the non-parametric Wilcoxon rank sum test. Normalisation of data was against the median of geNorm selected controls (MRPL19, PGK1, PPIA, TFRC and UBC) of least expression variation (see Materials and methods section). ${ }^{* *} P<0.001$.

$(n=50)$. We examined PRMT6 mRNA levels by Q-RT-PCR, and observed significantly decreased PRMT6 mRNA expression in the IDC breast cancer cohort relative to normal breast (Fig. 6A). Furthermore, the expression of the (in vitro identified) PRMT6-dependent breast cancer-related genes (Table 2) (SRA stem-loop interacting RNA binding protein: SLIRP; endothelial differentiation-related factor 1: EDF1; FKBP4, HIPK3, NCOA4, NAD(P)H dehydrogenase, quinone 1: NQO1; proliferationassociated 2G4: PA2G4; protein kinase C, delta: PRKCD; PTEN and tudor domain containing 3: TRIP13) demonstrated significant and differential expression, as observed after PRMT6 mRNA attenuation in breast cancer cell lines (Fig. 6B). The only 
Table 2 Genes identified as being transcriptionally regulated by PRMT6 and examined in human breast tissue: cancer relevance

\begin{tabular}{lll}
\hline Gene symbol & Cancer relevance & Reference \\
\hline AKR1C2; AKR1C1 & Progesterone metabolism & Ji et al. (2004) \\
SLIRP & Nuclear receptor co-repressor & Hatchell et al. (2006) \\
EDF1 & Sensitivity to tamoxifen resistance & Mendes-Pereira et al. (2012) \\
FKBP4 & Breast cancer prediction marker & Yang et al. (2012) \\
HIPK3 & Resistance to Fas receptor-mediated apoptosis & Curtin \& Cotter (2004) \\
NQ01 & Nitrosamine metabolism, dysregulated in nasopharyngeal carcinoma & Dodd et al. (2006) \\
PA2G4 & Inhibition of heregulin-mediated MCF-7 breast cancer cell growth & Zhang et al. (2008) \\
PRKCD & Pro-proliferative factor in oestrogen-dependent breast cancer cells & McCracken et al. (2003) \\
PTEN & Tumour suppressor gene & Chow \& Baker (2006) \\
TRIP13 & Interacts with thyroid hormone receptor, lung cancer marker & Kang et al. (2008) \\
\hline
\end{tabular}

exceptions were PTEN and TRIP13, which showed a significant yet opposite expression pattern in comparison with the in vitro results (see Fig. 2).

We then examined the four cancer-related genes we identified and validated to be alternatively spliced upon PRMT6 knockdown in MCF-7 and T-47D breast

A

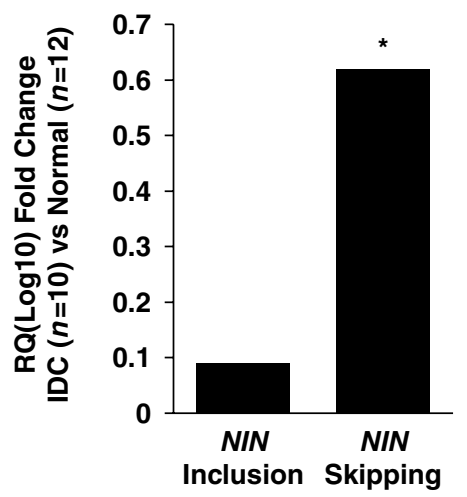

cancer cell lines. Examination of the IDC breast cancer cohort $(n=10)$ and normal breast tissue $(n=12)$ by Q-RT-PCR revealed changes in the exon usage of three of our previously identified alternatively spliced genes in the IDC cohort relative to normal breast tissue (Fig. 7A, B and C). We found increased NIN exon 19
B

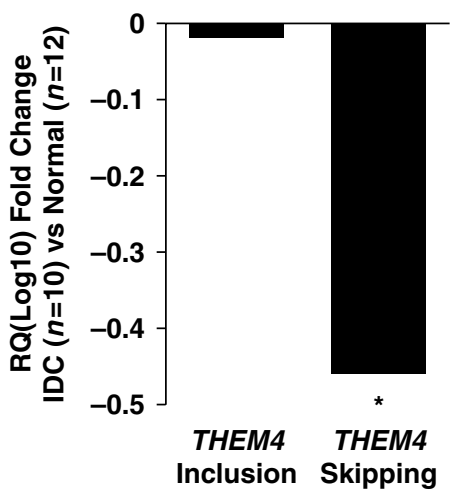

D

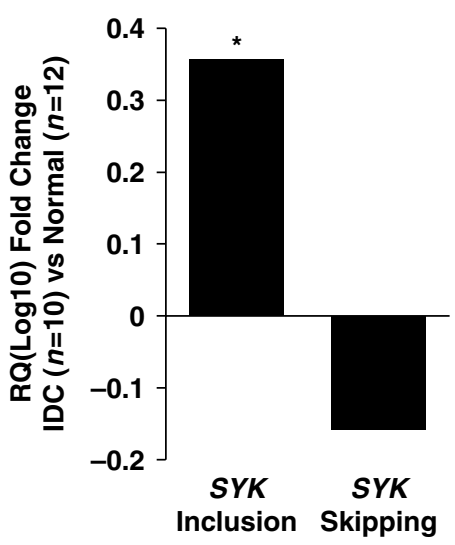

Figure 7 Expression of alternatively spliced transcripts in breast cancer. The expression of alternatively spliced transcripts for (A) NIN, (B) THEM4, (C) BCCIP and (D) SYK in invasive ductile carcinoma (IDC) human breast tissue samples ( $n=10)$ relative to normal human breast tissue $(n=12)$. Data are presented as relative quantification (RQ) (log10) of genes from IDC human breast tissue samples relative to normal human breast tissue. Statistical analysis was conducted with Integromics Statminer software package using the non-parametric Wilcoxon rank sum test. Normalisation of data was against the median of IPO 8 and TBP controls (see Materials and methods section). ${ }^{\star} P<0.05,{ }^{\star \star} P<0.01$. 
inclusion, decreased skipping of THEM4 exon 3, increased levels of the BCCIP $\beta$ isoform and no significant change in CTNND1 exon usage (data not shown). Our previous study (Harrison et al. 2010) demonstrated PRMT6-dependent alternative splicing of the breast cancer tumour suppressor SYK to be regulated by PRMT6 in breast cancer cell lines. In that study, we found reduction of PRMT6 in breast cancer cells resulted in an increase in the full-length $S Y K$ transcript (SYK-L) over the shorter SYK (SYK-S) transcript, which skips exon 7. Interestingly, we found similar increase in the $S Y K-L$ mRNA transcript expression in our IDC breast cancer samples (Fig. 7D).

\section{Expression of the PRMT6-dependent gene signature predicts disease outcome}

To investigate and expand upon the clinical relevance of our findings, we evaluated a PRMT6 transcriptionally regulated gene signature in the context of clinical outcome associations in three independent publically available breast cancer datasets with available clinical outcomes (van de Vijver et al. 2002, Desmedt et al. 2008, Miller et al. 2011). To determine whether PRMT6-dependent gene expression is associated with disease recurrence (RFS) and/or metastasis (DMFS), we utilised a PRMT6-dependent gene expression signature based on the 159 genes that were differentially expressed in a significant manner relative to controls ( $\geq 1.3$-fold, $P<0.01$; see Supplementary Table 1). PRMT6 gene expression signature scores were calculated for each patient tumour sample. The PRMT6-dependent gene expression signature is calculated as a signed average, with the sign of the PRMT6 gene expression signature score providing an indication of PRMT6 dysfunction (and by inference the level of PRMT6 expression). For example, a negative (i.e. low) PRMT6 gene expression signature score indicates that the genes comprising the PRMT6 gene expression signature and having a negative weight (i.e. the genes significantly down-regulated in the PRMT6 knockdown cells) are up-regulated relative to the PRMT6 signature genes having a positive weight (i.e. the genes significantly up-regulated in the PRMT6 knockdown cells). Accordingly, a negative or low PRMT6-dependent signature score is indicative of normal to high PRMT6 expression levels and a positive or high PRMT6-dependent signature score is indicative of PRMT6 dysfunction (i.e. low PRMT6 expression levels). In keeping with this notion, we observed a significant inverse correlation between the PRMT6 gene expression levels and PRMT6-dependent signature scores in the NKI and UPP breast cancer datasets
(Supplementary Figure 4A and B, see section on supplementary data given at the end of this article).

Patient samples were stratified according to their PRMT6-dependent gene signature scores (high scores $\geq 66 \%$ quantile; low scores $\leq 33 \%$ quantile). KaplanMeier survival analysis for RFS or DMFS (Fig. 8A, B and C, Supplementary Figures 5 and 6, see section on supplementary data given at the end of this article) clearly demonstrated that patients falling within the ER + subgroup and having low PRMT6 signature scores (i.e. indicative of normal PRMT6 function/ normal to high PRMT6 expression levels) exhibited significantly lower $(P<0.05$ in all cohorts analysed $)$ probabilities of RFS and metastasis-free survival when compared to patients falling within the ER+ subgroup and having high PRMT6 signature scores (i.e. indicative of PRMT6 dysfunction/low PRMT6 expression levels). Accordingly, the PRMT6dependent gene signature is significantly associated with clinical outcome in the ER + subgroup and suggests the PRMT6-dependent genetic program plays a significant role in breast cancer disease progression.

\section{Discussion}

We used Affymetrix exon array in combination with RNA interference technology to investigate the global effects of PRMT6 on transcription and alternative splicing in breast cancer cells and human breast cancer. Before this study, the role of PRMT6 in regulating transcription was controversial. We previously identified PRMT6 as a SHR co-activator capable of promoting the transcription of oestrogen- and progesterone-dependent genes (Harrison et al. 2010). However, earlier studies demonstrated a role for PRMT6 in repressing transcription by methylating H3R2 (Guccione et al. 2007, Hyllus et al. 2007, Iberg et al. 2008). In order to resolve this controversy, we examined the effects of knocking down PRMT6 on global transcription in breast cancer cells. We found that PRMT6 plays a dual role and can both co-activate and co-repress transcription. This is in agreement with a recent study in lung and bladder cell lines, in which PRMT6 functioned primarily as a transcriptional enhancer but could also repress transcription of a few genes (Yoshimatsu et al. 2011). The ability of PRMT6 to co-activate or co-repress transcription of a given gene, as demonstrated in our knockdown studies, may be due to direct or indirect effects. Regardless of the mode of action, our study highlights the fact that the PRMT6-dependent pathways do not exclusively modulate gene expression in a positive or negative fashion. Therefore, there is no conflict between the 


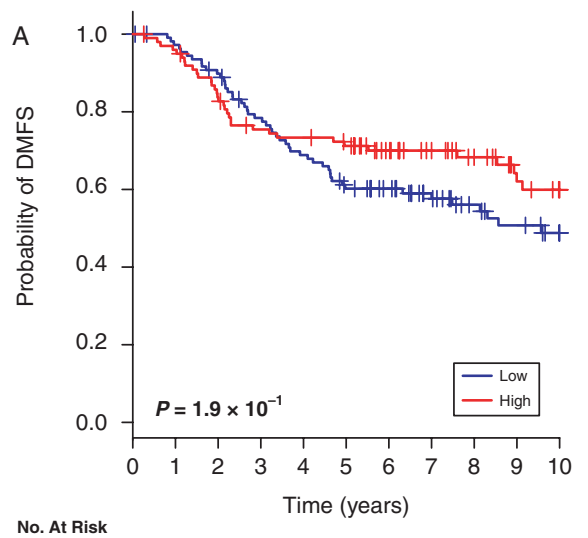

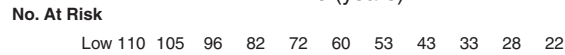

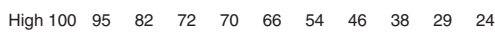

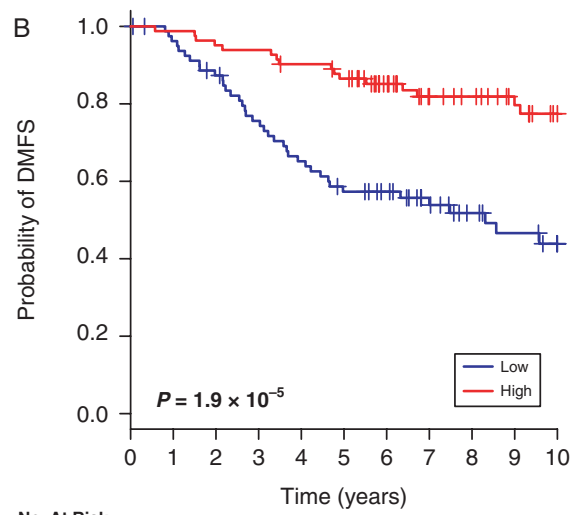
No. At Risk

$\begin{array}{llllllllllll}\text { Low } & 81 & 76 & 68 & 58 & 50 & 42 & 38 & 30 & 22 & 18 & 14\end{array}$ $\begin{array}{llllllllllll}\text { High } & 82 & 81 & 78 & 77 & 73 & 69 & 57 & 47 & 43 & 36 & 29\end{array}$

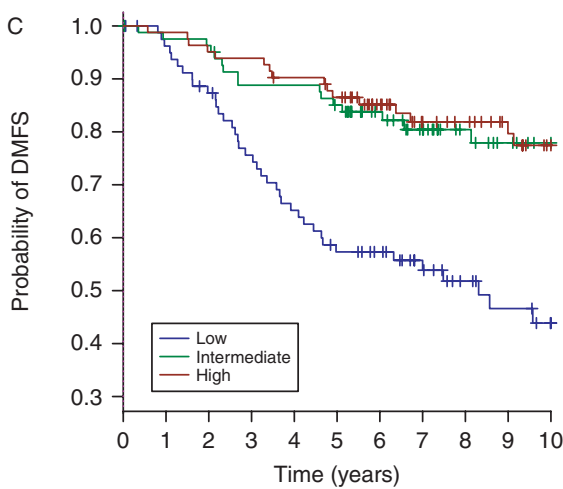
No. At Risk

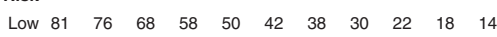
$\begin{array}{lllllllllllll}\text { Intermediate } & 82 & 79 & 78 & 71 & 71 & 67 & 52 & 43 & 32 & 27 & 23\end{array}$

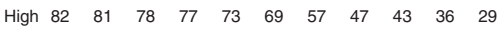

Figure 8 Long-term disease outcome based on PRMT6dependent transcriptional signature in breast cancer. Kaplan-Meier survival analysis of patients in the NKI breast cancer cohort exhibiting high and low PRMT6-dependent signature scores. Analysis was performed for (A) all subgroups and (B) patients falling within the ER+ subgroup. PRMT6-dependent signature scores were split according to their $33 \%$ and $66 \%$ quantiles. (C) Patients falling within the $\mathrm{ER}+$ subgroup $(P=3.4$ $\times 10^{-7}$ low vs high/intermediate). PRMT6-dependent signature scores were split according to their 33 and $66 \%$ quantiles. previous studies investigating the role of PRMT6 in gene expression, with the PRMT6-dependent transcriptional outcome likely to be gene dependent.

While the exact mechanism of PRMT6-dependent gene activation is still unknown, it has been shown that PRMT6 can methylate histone H4R3, a target that is also methylated by PRMT1 during oestrogen-induced gene activation (Strahl et al. 2001, Wagner et al. 2006, Hyllus et al. 2007). H4R3 methylation is read by tudor domain containing 3, an effector molecule that has transcriptional co-activator properties (Yang et al. 2010). It is possible that chromatin architecture at promoter/enhancer regions or previously laid down chromatin modifications determine whether the PRMT activity enhances or inhibits transcription.

Pathway analysis of PRMT6-dependent transcriptionally regulated genes indicates that PRMT6 may play a role in many cellular pathways, including those implicated in tumourigenesis. PRMT6 has been shown to be required for the G1-S transition and proliferation of lung and bladder cancer cells and is aberrantly regulated in a number of cancers, including breast cancer (Yoshimatsu et al. 2011). In addition, we have previously demonstrated that PRMT6 is required for maximal oestrogen-stimulated proliferation of MCF-7 breast cancer cells (Harrison et al. 2010).

We validated the results from the exon array by examining PRMT6-mediated changes in gene expression of eight genes, six of which have known roles in cancer. NCOA4, YWHAE, ARL6IP1 and MINA are overexpressed in a number of different cancers $(\mathrm{Hu}$ et al. 2004, Teye et al. 2004, Cimino et al. 2008, Peng et al. 2008, Guo et al. 2010). The up-regulation of YWHAE in breast cancer is associated with diseasefree and overall survival, and therefore, the downregulation of YWHAE from reduced PRMT6 levels may be unfavourable in relation to breast cancer prognosis (Cimino et al. 2008). Conversely, downregulation of PRMT6 expression and corresponding low expression of ARL6IP1 and MINA may be favourable to decreased breast cancer growth.

In our study, PTEN and IGFBP3 expression were increased when we knocked down PRMT6 in the MCF-7 and T-47D cell lines. PTEN is a tumour suppressor gene that inhibits the PI3K pathway (Chow \& Baker 2006). Loss of PTEN leads to the activation of a number of kinases and consequent cell cycle progression (Gera et al. 2004, Guertin \& Sabatini 2005). IGFBP3 has an antiproliferative effect and induces apoptosis in breast cancer cells (Kim et al. 2004, 2010). This indicates that lower PRMT6 expression may result in increased PTEN and IGFBP3 expression, decreased cell cycle progression and increased breast cancer cell apoptosis. 
We have demonstrated that PRMT6 has roles in steroid hormone-dependent transcription (Harrison et al. 2010), and pathway analysis demonstrated that PRMT6 regulates the transcription of genes involved in endocrine system development and function. HIPK3 and NCOA4 are AR co-activators (Yeh \& Chang 1996, Moilanen et al. 1998), and FKBP4 promotes binding of hormone to the GR (Riggs et al. 2003). This indicates that in addition to co-activation of steroid hormonedependent genes, PRMT6 is involved in the expression of genes that promote steroid hormone signalling. The reduction of PRMT6 in the MCF-7 and T47D $\mathrm{ER}+$ cell lines results in a decrease in HIPK3 and NCOA4, two transcriptional co-activators primarily associated with androgen receptor (AR) signalling. Interestingly, AR signalling in ER + breast cancer is thought to be associated with favourable outcomes (Castellano et al. 2010, Park et al. 2011), and the reduction of HIPK3 and NCOA4 may have a negative effect in relation to AR signalling and outcomes in $\mathrm{ER}+$ breast cancer. In contrast, reduction of the fulllength isoform of NCOA4 in MCF-7 cells is associated with increased breast cancer metastasis (Wu et al. 2011), while knockdown of NCOA4 in MCF-7 cells results in decreased cell proliferation (Hua et al. 2008). The exact role NCOA4 plays in breast cancer is not fully defined and requires further investigation.

PRMT6 functions as a tertiary co-regulator, and from our microarray data, is able to modulate the expression of a large number of genes. In the context of breast cancer, the expression of several of the examined PRMT6-regulated oncogene/tumour suppressor genes indicates that reduction of PRMT6 and corresponding PRMT6-dependent gene changes may be involved in breast carcinogenesis.

Analysis of the exon array data identified 449 genes that are alternatively spliced in response to PRMT6. Of these genes, 121 were also regulated transcriptionally by PRMT6. Therefore, PRMT6 can have a major impact on the function of particular genes by simultaneously regulating both their expression levels and final protein composition. However, the fact that not all genes overlap in the two groups demonstrates that PRMT6-regulated transcription and alternative splicing are not necessarily inter-dependent processes.

Pathway analysis of the alternatively spliced genes regulated by PRMT6 indicates that PRMT6 may play roles in cancer, gene expression, cell death and cell cycle progression. Therefore, it would appear that dysregulation of PRMT6 may lead to tumourigenesis by causing aberrant alternative splicing as well as by deregulating transcription. NIN plays a key role in centrosome function, which may be regulated by
PRMT6-directed alternative splicing. PRMT6 promotes skipping of NIN exon 19, which removes a region important in the centrosomal targeting ability of NIN. Therefore, removal of this region is likely to interfere with NIN chromosomal targeting and $\gamma$-tubulin binding, resulting in mitotic abnormalities (Abal et al. 2002, Dammermann \& Merdes 2002, Stillwell et al. 2004, Lin et al. 2006).

CTNND1 exon 20 encodes a functional leucine-rich nuclear export signal (Keirsebilck et al. 1998, van Hengel et al. 1999), and so PRMT6-directed inclusion of this exon will lead to cytoplasmic CTNND1. CTNND1 is often located in the cytoplasm but rarely in the nucleus of tumour cells, and cytoplasmic CTNND1 has been linked to a more invasive form of breast cancer (Sarrio et al. 2004, Shibata et al. 2004, Soubry et al. 2005). Whilst the metastatic-promoting role of cytoplasmic CTNND1 is unknown, it is possibly due to its binding to microtubules and/or activation of Rac (Franz \& Ridley 2004). In addition, CTNND1 may sequester Kaiso in the cytoplasm, preventing Kaiso-regulated repression of tumour suppressor genes (van Roy \& McCrea 2005), leading to a more oncogenic version of CTNND1 being expressed.

THEM4 is a mitochondrial protein that sensitises cells to apoptosis by binding to heat-shock protein 70 and promoting caspase-3 cleavage (Parcellier et al. 2009, Piao et al. 2009). Currently, nothing is known about the effect of inclusion/skipping of exon 3 on THEM4 structure and activity. Considering the importance of THEM4 in the apoptotic pathway, this PRMT6regulated alternative splicing event is certainly worth further investigation in relation to breast cancer.

BCCIP is a BRCA2 and CDKN1A (Cip1/waf1/p21) interacting protein that plays an important role in cancer aetiology (Ono et al. 2000, Liu et al. 2001). BCCIP is alternatively spliced to form two isoforms $(-\alpha$ and $-\beta)$ and PRMT6 knockdown leads to the preferential production of BCCIP $\beta$ in breast cancer cells. Whilst both BCCIP isoforms inhibit cell growth by interacting with $\mathrm{p} 21, \mathrm{BCCIP} \beta$ has a much greater effect on cell growth inhibition, particularly in breast cancer cells (Meng et al. 2004).

We found that PRMT6 expression was substantially down-regulated in IDC compared with normal breast tissue. The recent study by Yoshimatsu et al. (2011) found PRMT6 to be significantly increased in $~ 33 \%$ of breast cancers. Knowing that breast cancer is not a homogeneous disease, it is not surprising that individual breast cancer tumours demonstrate dissimilar expression of PRMT6. Given our findings and that of Yoshimatsu et al.'s (2011), PRMT6 may be abnormally regulated up or down in breast cancer. Many of the 
genes we identified as transcriptionally regulated after attenuation of PRMT6 in MCF-7 and T-47D cells also demonstrated changes in expression in IDC breast cancer compared to normal breast tissue, including SLIRP, EDF1, FKBP4, HIPK3, NCOA4, NQO1, $P A 2 G 4, P R K C D$ and PTEN. Similarly, genes we have identified as alternatively spliced by PRMT6 in our array and previous studies (NIN, THEM4, BCCIP $\beta$ and $S Y K$ ) demonstrate changes in alternative splicing in IDC compared to normal breast tissue.

Through the identification of genes regulated by PRMT6 at the gene expression level, we were able to investigate the significance of a PRMT6-dependent gene expression signature in three published breast cancer cohorts (Fig. 8A, B and C, Supplementary Figures 5 and 6). We found that low PRMT6 expression and the corresponding high PRMT6-dependent gene signature correlated with increased probability of relapse-free or metastasis-free survival in ER + breast cancer. This would indicate that the role of PRMT6 in breast cancer is associated with ER-mediated signalling but extends beyond $S H R$ transcriptional regulation.

PRMT6 can determine transcriptional gene regulation and alternative splicing decisions, including both exon retention and skipping. Therefore, PRMT6 can be considered a master modulator of gene expression, capable of determining both transcript abundance and protein structure. Our findings indicate that PRMT6 mRNA is aberrantly down-regulated in breast cancer and the genes we found regulated by PRMT6 in human breast cancer cells are overrepresented by genes involved in important aspects of carcinogenesis. In addition, our PRMT6-dependent gene signature is significantly associated with clinical outcome in ER + breast cancer and suggests that the PRMT6-dependent genetic program plays a significant role in breast cancer disease progression.

\section{Supplementary data}

This is linked to the online version of the paper at http://dx. doi.org/10.1530/ERC-12-0100.

\section{Declaration of interest}

D HDowhan and George E O Muscat are co-Chief Investigators on a 2012/2013 grant submission. All other authors declare that there is no conflict of interest that could be perceived as prejudicing the impartiality of the research reported.

\section{Funding}

This work was supported by a National Health and Medical Research Council of Australia (NHMRC) project grant
(Grant\#511153) to D H Dowhan. M J Harrison was the recipient of an ANZ Trustees $\mathrm{PhD}$ Medical Research scholarship. In addition, this research was supported by a grant from the National Breast Cancer Foundation (NBCF), Australia.

\section{Author contribution statement}

D H Dowhan conceived the study, performed breast tissue experimental studies, data analysis, participated the study design, coordination and manuscript preparation. M J Harrison participated the study design, performed cell line experimental studies, data analysis and manuscript preparation. N A Eriksson performed breast tissue RNA extraction, experimental studies and data analysis. P Bailey and M A Brown performed public data set mining, KaplanMeier survival analysis and data analysis. M A Pearen carried out PCA and Ingenuity analysis on the exon array data. P J Fuller, J W Funder, E R Simpson, P J Leedman, W D Tilley, C L Clarke and G E O Muscat provided breast tissue samples and were involved in the study, design and analysis of transcriptional profiling studies in breast cancer. P Bailey, P J Fuller, J W Funder, E R Simpson, P J Leedman, W D Tilley, M A Brown and C L Clarke assisted in manuscript preparation. G E O Muscat participated in the study design, coordination and manuscript preparation. All authors read and approved the final manuscript.

\section{Acknowledgements}

Breast cancers and normal tissues were provided by Australian Breast Cancer Tissue Bank (BCTB), which is supported by the NHMRC of Australia, the Cancer Institute NSW and the NCBF, or by the Victorian Cancer BioBank Australia, which is supported by the Victorian Government. BCTB tissues and samples were made available to researchers on a non-exclusive basis. Normal breast biopsies were obtained from the Susan G Komen for the Cure Tissue Bank at the IU Simon Cancer Center. The authors thank contributors to the Susan G Komen for the Cure Tissue Bank, including Indiana University, who collected the samples used in this study, as well as patients and their families, whose help and participation made this work possible. The authors would like to thank the Ramaciotti Centre for Gene Function Analysis (University of NSW), for RNA conversion, exon array hybridization and data collection and Dr Paul Leo for initial microarray analysis.

\section{References}

Abal M, Piel M, Bouckson-Castaing V, Mogensen M, Sibarita JB \& Bornens M 2002 Microtubule release from the centrosome in migrating cells. Journal of Cell Biology 159 731-737. (doi:10.1083/jcb.200207076)

Aletta JM \& Hu JC 2008 Protein arginine methylation in health and disease. Biotechnology Annual Review 14 203-224. (doi:10.1016/S1387-2656(08)00008-2) 
Barrett T, Suzek TO, Troup DB, Wilhite SE, Ngau WC, Ledoux P, Rudnev D, Lash AE, Fujibuchi W \& Edgar R 2005 NCBI GEO: mining millions of expression profiles database and tools. Nucleic Acids Research 33 D562-D566. (doi:10.1093/nar/gki022)

Bedford MT \& Clarke SG 2009 Protein arginine methylation in mammals: who, what, and why. Molecular Cell $\mathbf{3 3}$ 1-13. (doi:10.1016/j.molcel.2008.12.013)

Bedford MT \& Richard S 2005 Arginine methylation an emerging regulator of protein function. Molecular Cell 18 263-272. (doi:10.1016/j.molcel.2005.04.003)

Castellano I, Allia E, Accortanzo V, Vandone AM, Chiusa L, Arisio R, Durando A, Donadio M, Bussolati G, Coates AS et al. 2010 Androgen receptor expression is a significant prognostic factor in estrogen receptor positive breast cancers. Breast Cancer Research and Treatment 124 607-617. (doi:10.1007/s10549-010-0761-y)

Cheng D, Cote J, Shaaban S \& Bedford MT 2007 The arginine methyltransferase CARM1 regulates the coupling of transcription and mRNA processing. Molecular Cell 25 71-83. (doi:10.1016/j.molcel.2006.11.019)

Chow LM \& Baker SJ 2006 PTEN function in normal and neoplastic growth. Cancer Letters 241 184-196. (doi:10.1016/j.canlet.2005.11.042)

Cimino D, Fuso L, Sfiligoi C, Biglia N, Ponzone R, Maggiorotto F, Russo G, Cicatiello L, Weisz A, Taverna D et al. 2008 Identification of new genes associated with breast cancer progression by gene expression analysis of predefined sets of neoplastic tissues. International Journal of Cancer 123 1327-1338. (doi:10.1002/ijc.23660)

Curtin JF \& Cotter TG 2004 JNK regulates HIPK3 expression and promotes resistance to Fas-mediated apoptosis in DU 145 prostate carcinoma cells. Journal of Biological Chemistry 279 17090-17100. (doi:10.1074/jbc.M307629200)

Dammermann A \& Merdes A 2002 Assembly of centrosomal proteins and microtubule organization depends on PCM-1. Journal of Cell Biology 159 255-266. (doi:10.1083/jcb.200204023)

Desmedt C, Haibe-Kains B, Wirapati P, Buyse M, Larsimont D, Bontempi G, Delorenzi M, Piccart M \& Sotiriou C 2008 Biological processes associated with breast cancer clinical outcome depend on the molecular subtypes. Clinical Cancer Research 14 5158-5165. (doi:10.1158/1078-0432.CCR-07-4756)

Dodd LE, Sengupta S, Chen IH, den Boon JA, Cheng YJ, Westra W, Newton MA, Mittl BF, McShane L, Chen CJ et al. 2006 Genes involved in DNA repair and nitrosamine metabolism and those located on chromosome 14q32 are dysregulated in nasopharyngeal carcinoma. Cancer Epidemiology, Biomarkers \& Prevention 15 2216-2225. (doi:10.1158/1055-9965.EPI-06-0455)

Dutertre M, Lacroix-Triki M, Driouch K, de la Grange P, Gratadou L, Beck S, Millevoi S, Tazi J, Lidereau R, Vagner S et al. 2010 Exon-based clustering of murine breast tumor transcriptomes reveals alternative exons whose expression is associated with metastasis. Cancer Research 70 896-905. (doi:10.1158/0008-5472.CAN-09-2703)
Eng C 2003 PTEN: one gene, many syndromes. Human Mutation 22 183-198. (doi:10.1002/humu.10257)

Franz CM \& Ridley AJ 2004 p120 catenin associates with microtubules: inverse relationship between microtubule binding and Rho GTPase regulation. Journal of Biological Chemistry 279 6588-6594. (doi:10.1074/jbc.M312812200)

Gera JF, Mellinghoff IK, Shi Y, Rettig MB, Tran C, Hsu JH, Sawyers CL \& Lichtenstein AK 2004 AKT activity determines sensitivity to mammalian target of rapamycin (mTOR) inhibitors by regulating cyclin D1 and c-myc expression. Journal of Biological Chemistry 279 2737-2746. (doi:10.1074/jbc.M309999200)

Guccione E, Bassi C, Casadio F, Martinato F, Cesaroni M, Schuchlautz H, Luscher B \& Amati B 2007 Methylation of histone H3R2 by PRMT6 and H3K4 by an MLL complex are mutually exclusive. Nature 449 933-937. (doi:10.1038/nature06166)

Guertin DA \& Sabatini DM 2005 An expanding role for mTOR in cancer. Trends in Molecular Medicine 11 353-361. (doi:10.1016/j.molmed.2005.06.007)

Guo F, Liu Y, Li Y \& Li G 2010 Inhibition of ADPribosylation factor-like 6 interacting protein 1 suppresses proliferation and reduces tumor cell invasion in CaSki human cervical cancer cells. Molecular Biology Reports 37 3819-3825. (doi:10.1007/s11033-010-0037-y)

Haibe-Kains B 2009 Identification and Assessment of Gene Signatures in Human Breast Cancer. PhD thesis. Universite Libre de Bruxelles.

Haibe-Kains B, Desmedt C, Sotiriou C \& Bontempi G 2008 A comparative study of survival models for breast cancer prognostication based on microarray data: does a single gene beat them all? Bioinformatics 24 2200-2208. (doi:10.1093/bioinformatics/btn374)

Haibe-Kains B, Bontempi B, Quackenbush J \& Sotiriou C $2010 a$ genefu: Relevant Functions for Gene Expression Analysis, Especially in Breast Cancer. R package version 1.0.9. http://CRAN.R-project.org/package=genefu.

Haibe-Kains B, Sotiriou C \& Bontempi G $2010 b$ survcomp: Performance Assessment and Comparison for Survival Analysis. R package version 1.1.6. http://CRAN.R-project. org/package $=$ survcomp.

Harrison MJ, Tang YH \& Dowhan DH 2010 Protein arginine methyltransferase 6 regulates multiple aspects of gene expression. Nucleic Acids Research 38 2201-2216. (doi:10.1093/nar/gkp1203)

Hatchell EC, Colley SM, Beveridge DJ, Epis MR, Stuart LM, Giles KM, Redfern AD, Miles LE, Barker A, MacDonald LM et al. 2006 SLIRP, a small SRA binding protein, is a nuclear receptor corepressor. Molecular Cell 22 657-668. (doi:10.1016/j.molcel.2006.05.024)

van Hengel J, Vanhoenacker P, Staes K \& van Roy F 1999 Nuclear localization of the p120(ctn) Armadillo-like catenin is counteracted by a nuclear export signal and by E-cadherin expression. PNAS 96 7980-7985. (doi:10.1073/pnas.96.14.7980)

Hu YC, Yeh S, Yeh SD, Sampson ER, Huang J, Li P, Hsu CL, Ting HJ, Lin HK, Wang L et al. 2004 Functional domain 
and motif analyses of androgen receptor coregulator ARA70 and its differential expression in prostate cancer. Journal of Biological Chemistry 279 33438-33446. (doi:10.1074/jbc.M401781200)

Hua S, Kallen CB, Dhar R, Baquero MT, Mason CE, Russell BA, Shah PK, Liu J, Khramtsov A, Tretiakova MS et al. 2008 Genomic analysis of estrogen cascade reveals histone variant H2A.Z associated with breast cancer progression. Molecular Systems Biology 4188. (doi:10.1038/msb.2008.25)

Hyllus D, Stein C, Schnabel K, Schiltz E, Imhof A, Dou Y, Hsieh J \& Bauer UM 2007 PRMT6-mediated methylation of R2 in histone H3 antagonizes H3 K4 trimethylation. Genes and Development 21 3369-3380. (doi:10.1101/gad.447007)

Iberg AN, Espejo A, Cheng D, Kim D, Michaud-Levesque J, Richard S \& Bedford MT 2008 Arginine methylation of the histone $\mathrm{H} 3$ tail impedes effector binding. Journal of Biological Chemistry 283 3006-3010. (doi:10.1074/jbc. C700192200)

Ingermann AR, Yang YF, Han J, Mikami A, Garza AE, Mohanraj L, Fan L, Idowu M, Ware JL, Kim HS et al. 2010 Identification of a novel cell death receptor mediating IGFBP-3-induced anti-tumor effects in breast and prostate cancer. Journal of Biological Chemistry 285 30233-30246. (doi:10.1074/jbc.M110.122226)

Ji Q, Aoyama C, Nien YD, Liu PI, Chen PK, Chang L, Stanczyk FZ \& Stolz A 2004 Selective loss of AKR1C1 and AKR1C2 in breast cancer and their potential effect on progesterone signaling. Cancer Research 64 7610-7617. (doi:10.1158/0008-5472.CAN-04-1608)

Kang JU, Koo SH, Kwon KC, Park JW \& Kim JM 2008 Gain at chromosomal region 5p15.33, containing TERT, is the most frequent genetic event in early stages of non-small cell lung cancer. Cancer Genetics and Cytogenetics 182 1-11. (doi:10.1016/j.cancergencyto.2007.12.004)

Keirsebilck A, Bonne S, Staes K, van Hengel J, Nollet F, Reynolds A \& van Roy F 1998 Molecular cloning of the human p120ctn catenin gene (CTNND1): expression of multiple alternatively spliced isoforms. Genomics $\mathbf{5 0}$ 129-146. (doi:10.1006/geno.1998.5325)

Kim HS, Ingermann AR, Tsubaki J, Twigg SM, Walker GE \& Oh Y 2004 Insulin-like growth factor-binding protein 3 induces caspase-dependent apoptosis through a death receptor-mediated pathway in MCF-7 human breast cancer cells. Cancer Research 64 2229-2237. (doi:10.1158/0008-5472.CAN-03-1675)

Kim HS, Lee WJ, Lee SW, Chae HW, Kim DH \& Oh Y 2010 Insulin-like growth factor binding protein-3 induces G1 cell cycle arrest with inhibition of cyclin-dependent kinase 2 and 4 in MCF-7 human breast cancer cells. Hormone and Metabolic Research 42 165-172. (doi:10.1055/s-0029-1243190)

Lee YH \& Stallcup MR 2009 Minireview: protein arginine methylation of nonhistone proteins in transcriptional regulation. Molecular Endocrinology 23 425-433. (doi:10.1210/me.2008-0380)
Lin CC, Cheng TS, Hsu CM, Wu CH, Chang LS, Shen ZS, Yeh HM, Chang LK, Howng SL \& Hong YR 2006 Characterization and functional aspects of human ninein isoforms that regulated by centrosomal targeting signals and evidence for docking sites to direct gamma-tubulin. Cell Cycle 5 2517-2527. (doi:10.4161/cc.5.21.3404)

Liu J, Yuan Y, Huan J \& Shen Z 2001 Inhibition of breast and brain cancer cell growth by BCCIP $\alpha$, an evolutionarily conserved nuclear protein that interacts with BRCA2. Oncogene 20 336-345. (doi:10.1038/sj.onc.1204098)

McCracken MA, Miraglia LJ, McKay RA \& Strobl JS 2003 Protein kinase $\mathrm{C}$ delta is a prosurvival factor in human breast tumor cell lines. Molecular Cancer Therapeutics 2 273-281.

Mendes-Pereira AM, Sims D, Dexter T, Fenwick K, Assiotis I, Kozarewa I, Mitsopoulos C, Hakas J, Zvelebil M, Lord CJ et al. 2012 Genome-wide functional screen identifies a compendium of genes affecting sensitivity to tamoxifen. PNAS 109 2730-2735. (doi:10.1073/pnas.1018872108)

Meng X, Liu J \& Shen Z 2004 Inhibition of G1 to S cell cycle progression by BCCIP $\beta$. Cell Cycle 3 343-348.

Miller TW, Balko JM, Ghazoui Z, Dunbier A, Anderson H, Dowsett M, Gonzalez-Angulo AM, Mills GB, Miller WR, $\mathrm{Wu} \mathrm{H}$ et al. 2011 A gene expression signature from human breast cancer cells with acquired hormone independence identifies MYC as a mediator of antiestrogen resistance. Clinical Cancer Research 17 2024-2034. (doi:10.1158/1078-0432.CCR-10-2567)

Moilanen AM, Karvonen U, Poukka H, Janne OA \& Palvimo JJ 1998 Activation of androgen receptor function by a novel nuclear protein kinase. Molecular Biology of the Cell 9 2527-2543.

Myers SA, Eriksson N, Burow R, Wang SC \& Muscat GEO $2009 \beta$-Adrenergic signaling regulates NR4A nuclear receptor and metabolic gene expression in multiple tissues. Molecular and Cellular Endocrinology 309 101-108. (doi:10.1016/j.mce.2009.05.006)

Ono T, Kitaura H, Ugai H, Murata T, Yokoyama KK, Iguchi-Ariga SM \& Ariga H 2000 TOK-1, a novel p21Cip1-binding protein that cooperatively enhances p21-dependent inhibitory activity toward CDK2 kinase. Journal of Biological Chemistry 275 31145-31154. (doi:10.1074/jbc.M003031200)

Paik WK \& Kim S 1967 Enzymatic methylation of protein fractions from calf thymus nuclei. Biochemical and

Biophysical Research Communications 29 14-20. (doi:10.1016/0006-291X(67)90533-5)

Parcellier A, Tintignac LA, Zhuravleva E, Cron P, Schenk S, Bozulic L \& Hemmings BA 2009 Carboxy-terminal modulator protein (CTMP) is a mitochondrial protein that sensitizes cells to apoptosis. Cellular Signalling 21 639-650. (doi:10.1016/j.cellsig.2009.01.016)

Park S, Koo JS, Kim MS, Park HS, Lee JS, Lee JS, Kim SI, Park BW \& Lee KS 2011 Androgen receptor expression is significantly associated with better outcomes in estrogen receptor-positive breast cancers. Annals of Oncology 22 1755-1762. (doi:10.1093/annonc/mdq678) 
Peng Y, Li CX, Chen F, Wang Z, Ligr M, Melamed J, Wei J, Gerald W, Pagano M, Garabedian MJ et al. 2008 Stimulation of prostate cancer cellular proliferation and invasion by the androgen receptor co-activator ARA70. American Journal of Pathology 172 225-235. (doi:10.2353/ajpath.2008.070065)

Piao L, Li Y, Yang KJ, Park KA, Byun HS, Won M, Hong J, Kim JL, Kweon GR, Hur GM et al. 2009 Heat shock protein 70-mediated sensitization of cells to apoptosis by carboxyl-terminal modulator protein. BMC Cell Biology 10 53. (doi:10.1186/1471-2121-10-53)

Raichur S, Fitzsimmons RL, Myers SA, Pearen MA, Lau P, Eriksson N, Wang SM \& Muscat GEO 2010 Identification and validation of the pathways and functions regulated by the orphan nuclear receptor, ROR $\alpha 1$, in skeletal muscle. Nucleic Acids Research 38 4296-4312. (doi:10.1093/nar/gkq180)

Riggs DL, Roberts PJ, Chirillo SC, Cheung-Flynn J, Prapapanich V, Ratajczak T, Gaber R, Picard D \& Smith DF 2003 The Hsp90-binding peptidylprolyl isomerase FKBP52 potentiates glucocorticoid signaling in vivo. EMBO Journal 22 1158-1167. (doi:10.1093/emboj/cdg108)

van Roy FM \& McCrea PD 2005 A role for Kaiso-p120ctn complexes in cancer? Nature Reviews. Cancer 5 956-964. (doi:10.1038/nrc1752)

Sarrio D, Perez-Mies B, Hardisson D, Moreno-Bueno G, Suarez A, Cano A, Martin-Perez J, Gamallo C \& Palacios J 2004 Cytoplasmic localization of p120ctn and E-cadherin loss characterize lobular breast carcinoma from preinvasive to metastatic lesions. Oncogene $\mathbf{2 3}$ 3272-3283. (doi:10.1038/sj.onc.1207439)

Shibata T, Kokubu A, Sekine S, Kanai Y \& Hirohashi S 2004 Cytoplasmic p120ctn regulates the invasive phenotypes of E-cadherin-deficient breast cancer. American Journal of Pathology 164 2269-2278. (doi:10.1016/S00029440(10)63783-2)

Sotiriou C, Wirapati P, Loi S, Harris A, Fox S, Smeds J, Nordgren H, Farmer P, Praz V, Haibe-Kains B et al. 2006 Gene expression profiling in breast cancer: understanding the molecular basis of histologic grade to improve prognosis. Journal of the National Cancer Institute 98 262-272. (doi:10.1093/jnci/djj052)

Soubry A, van Hengel J, Parthoens E, Colpaert C, Van Marck E, Waltregny D, Reynolds AB \& van Roy F 2005 Expression and nuclear location of the transcriptional repressor Kaiso is regulated by the tumor microenvironment. Cancer Research 65 2224-2233. (doi:10.1158/0008-5472.CAN-04-2020)

Stillwell EE, Zhou J \& Joshi HC 2004 Human ninein is a centrosomal autoantigen recognized by CREST patient sera and plays a regulatory role in microtubule nucleation. Cell Cycle 3 923-930. (doi:10.4161/cc.3.7.947)

Strahl BD, Briggs SD, Brame CJ, Caldwell JA, Koh SS, Ma H, Cook RG, Shabanowitz J, Hunt DF, Stallcup MR et al. 2001 Methylation of histone $\mathrm{H} 4$ at arginine 3 occurs in vivo and is mediated by the nuclear receptor coactivator PRMT1. Current Biology 11 996-1000. (doi:10.1016/ S0960-9822(01)00294-9)
Teye K, Tsuneoka M, Arima N, Koda Y, Nakamura Y, Ueta Y, Shirouzu K \& Kimura H 2004 Increased expression of a Myc target gene Mina53 in human colon cancer. American Journal of Pathology 164 205-216. (doi:10.1016/S0002-9440(10)63111-2)

Teyssier C, Le RM, Sentis S, Jalaguier S, Corbo L \& Cavailles V 2010 Protein arginine methylation in estrogen signaling and estrogen-related cancers. Trends in Endocrinology and Metabolism 21 181-189. (doi:10.1016/j.tem.2009.11.002)

van de Vijver MJ, He YD, van't Veer LJ, Dai H, Hart AA, Voskuil DW, Schreiber GJ, Peterse JL, Roberts C, Marton MJ et al. 2002 A gene-expression signature as a predictor of survival in breast cancer. New England Journal of Medicine 347 1999-2009. (doi:10.1056/ NEJMoa021967)

Wagner S, Weber S, Kleinschmidt MA, Nagata K \& Bauer UM 2006 SET-mediated promoter hypoacetylation is a prerequisite for coactivation of the estrogen-responsive pS2 gene by PRMT1. Journal of Biological Chemistry 281 27242-27250. (doi:10.1074/jbc.M605172200)

Wu X, Chen F, Sahin A, Albarracin C, Pei Z, Zou X, Singh B, Xu R, Daniels G, Li Y et al. 2011 Distinct function of androgen receptor coactivator ARA70 $\alpha$ and ARA70 $\beta$ in mammary gland development, and in breast cancer. Breast Cancer Research and Treatment 128 391-400. (doi:10.1007/s10549-010-1131-5)

Yang Y, Lu Y, Espejo A, Wu J, Xu W, Liang S \& Bedford MT 2010 TDRD3 is an effector molecule for arginine-methylated histone marks. Molecular Cell $\mathbf{4 0}$ 1016-1023. (doi:10.1016/j.molcel.2010.11.024)

Yang WS, Moon HG, Kim HS, Choi EJ, Yu MH, Noh DY \& Lee C 2012 Proteomic approach reveals FKBP4 and S100A9 as potential prediction markers of therapeutic response to neoadjuvant chemotherapy in patients with breast cancer. Journal of Proteome Research 11 1078-1088. (doi:10.1021/pr2008187)

Yeh S \& Chang C 1996 Cloning and characterization of a specific coactivator, ARA70, for the androgen receptor in human prostate cells. PNAS 93 5517-5521. (doi:10.1073/ pnas.93.11.5517)

Yoshimatsu M, Toyokawa G, Hayami S, Unoki M, Tsunoda T, Field HI, Kelly JD, Neal DE, Maehara Y, Ponder BA et al. 2011 Dysregulation of PRMT1 and PRMT6, type I arginine methyltransferases, is involved in various types of human cancers. International Journal of Cancer 128 562-573. (doi:10.1002/ijc.25366)

Zhang Y, Akinmade D \& Hamburger AW 2008 Inhibition of heregulin mediated MCF-7 breast cancer cell growth by the ErbB3 binding protein EBP1. Cancer Letters 265 298-306. (doi:10.1016/j.canlet.2008.02.024)

Received in final form 22 May 2012

Accepted 6 June 2012

Made available online as an Accepted Preprint 6 June 2012 